\title{
MicroRNA-361-5p induces hepatocellular carcinoma cell apoptosis and enhances drug sensitivity by targeting MAP3K9
}

\author{
QINGBO REN $^{1 *}$, XIANGMEI XIAO ${ }^{2 *}$, XIAOQIAN LENG ${ }^{2}$, QI ZHANG $^{2}$, \\ $\mathrm{XUE} \mathrm{ZHOU}^{2}$, ZIYUAN REN ${ }^{3}$ and HANG XIAO ${ }^{4}$
}

\author{
${ }^{1}$ Department of Clinical Laboratory, Qingdao Sixth People's Hospital, Qingdao, Shandong 266033; \\ ${ }^{2}$ Department of Clinical Laboratory, Qingdao Fifth People's Hospital; ${ }^{3}$ Medical College of Cheeloo, \\ Shandong University, Qingdao, Shandong 266000; ${ }^{4}$ Department of General Internal Medicine, \\ Qingdao Sixth People's Hospital, Qingdao, Shandong 266033, P.R. China
}

Received August 5, 2020; Accepted December 22, 2020

DOI: $10.3892 / \mathrm{etm} .2021 .10006$

\begin{abstract}
MicroRNAs (miRs) participate in the development of several cancers. miR-361-5p suppresses the proliferation of hepatocellular carcinoma (HCC) cells. However, its function and potential underlying mechanism of action in the chemoresistance of HCC remains unknown. Therefore, cisplatin (DDP)-resistant HCC cells were used to study the role and potential mechanism of action of miR-361-5p in HCC resistance to chemotherapy. TargetScan software and dual-luciferase reporter assays were used to determine whether MAPK kinase kinase 9 (MAP3K9) is a target gene of miR-361-5p. Subsequently, reverse transcription-quantitative PCR and western blot analyses demonstrated that miR-361-5p mimic decreased MAP3K9 expression levels in Huh7 cells and this change was reversed by transfection with the MAP3K9-plasmid. In addition, compared with THLE-2 cells, miR-361-5p was downregulated, while MAP3K9 was upregulated in Huh7 cells. MAP3K9 also reversed the miR-361-5p-induced HCC cell apoptosis. A DDP-resistant cell line, Huh7/DDP, was established and MTT analysis revealed that the $\mathrm{IC}_{50}$ value of DDP treatment in Huh7/DDP cells was higher compared with that in Huh7 cells. miR-361-5p expression was lower in Huh7/DDP cells compared with that in Huh7 cells. Similarly, miR-361-5p downregulated the expression levels of MAP3K9 in Huh7/DDP cells. Furthermore, MAP3K9 reversed miR-361-5p-induced sensitivity of Huh7/DDP cells to DDP and miR-361-5p induced Huh7/DDP cell apoptosis. Therefore, the findings of the present study demonstrated that
\end{abstract}

Correspondence to: Dr Hang Xiao, Department of General Internal Medicine, Qingdao Sixth People's Hospital, 9 Fushun Road, Qingdao, Shandong 266033, P.R. China

E-mail: re654787@163.com

${ }^{*}$ Contributed equally

Key words: Huh7/DDP cells, mitogen-activated protein kinase kinase kinase 9, microRNA-361-5p, hepatocellular carcinoma the miR-361-5p/MAP3K9 axis may serve as a new potential biomarker and therapeutic target for DDP-resistant HCC.

\section{Introduction}

Hepatocellular carcinoma ( $\mathrm{HCC}$ ), one of the most common malignant tumor types in China originates from hepatocytes or hepatic progenitor cells. There are obvious regional differences in the distribution of HCC $(1,2)$. The main characteristics of advanced liver cancer include liver pain, keratitis, jaundice and ascites (3). Recent data indicate that the cases of liver cancer have increased to one million annually, of whom 700,000 patients will eventually succumb to the disease $(4,5)$. Previously, the highest incidence of liver cancer was observed in the age group of 45-65 years; however, the incidence of liver cancer is exhibiting an increasing trend among younger individuals (6). At present, there are several clinical treatment options for liver cancer, including tumor resection, chemotherapy and radiotherapy (7). However, the 5-year survival rate for patients with liver cancer is not satisfactory and a number of patients exhibit drug resistance (8). Cisplatin (DDP) is a common chemotherapeutic drug that can be used to treat a variety of cancer types $(9,10)$. However, DDP-based chemotherapy is usually limited by intrinsic and acquired resistance, which leads to chemotherapy failure. Therefore, it is imperative to identify new targets in order to improve the prognosis for patients with $\operatorname{HCC}(11,12)$.

MicroRNAs (miRNAs/miRs) are a group of RNAs that are endogenous, non-protein coding, single-stranded small molecules, which are 18-24 nucleotides in length and regulate downstream gene expression at the post-transcriptional level (13-15). miRNAs participate in a number of biological processes and changes in the expression and function of miRNAs are implicated in several diseases (16). Accumulating evidence has demonstrated that multiple abnormally expressed miRNAs are associated with the development of HCC, including miR-3174, miR-383, miR-361-5p and miR-330-5p (17-21). Wang et al (18) demonstrated that miR-383 suppresses cell proliferation and induces apoptosis. A previous study has demonstrated that miR-3174 is highly expressed in HCC (17). miR-361-5p has been reported to be downregulated in HCC (20) and Sun et al (19) reported that 
miR-361-5p inhibits HCC cell growth. However, the function of miR-361-5p in the chemoresistance of HCC and the potential molecular mechanism of action remain unknown. In the present study, DDP-resistant HCC cells were used to study the function and potential mechanism of action of miR-361-5p.

MAPK kinase kinase 9 (MAP3K9) is an upstream activator of the MAPK kinase/JNK signaling pathway, which plays critical roles in the regulation of cell apoptosis (22). The MAPK family consists of three subgroups: ERK1/2, stress-activated protein kinase/JNK and p38 MAPK (23). Furthermore, Nie et al (24) demonstrated that MAP3K9 is a target gene of miR-148b and Zhao et al (25) indicated that there is an interaction site between MAP3K9 and miR-1247. In the present study, bioinformatics software analysis was used to determine whether miR-361-5p and MAP3K9 have direct binding sites, as well as to elucidate whether miR-361-5p regulates cell apoptosis in HCC by mediating changes in MAP3K9 expression.

\section{Materials and methods}

Cell line acquirement and culture. Human normal hepatocytes (THLE-2) and the human HCC cell line Huh7 were obtained from American Tissue Culture Collection. THLE-2 and Huh7 cells were cultured in DMEM (Corning, Inc.) supplemented with 10\% FBS (Gibco; Thermo Fisher Scientific, Inc.) and incubated at $37^{\circ} \mathrm{C}$ in a $5 \% \mathrm{CO}_{2}$ incubator. A DDP-resistant cell line, Huh7/DDP, was established by exposing parental Huh7cells to increasing concentrations of DDP for 1 year, as previously described (26). Huh7 or Huh7/DDP cells were transfected with $100 \mathrm{nM}$ mimic control (5'-UUCUCCGAACGUGUCACGUTT-3'; Shanghai GenePharma Co., Ltd.), $100 \mathrm{nM}$ miR-361-5p mimic (5'-ACGCCUGGAGAUUCUGAUAAUU-3'; Shanghai GenePharma Co.,Ltd.), $1 \mu$ g control-plasmid (cat. no. sc-437275; Santa Cruz Biotechnology, Inc.), $1 \mu \mathrm{g}$ MAP3K9-plasmid (cat. no. sc-410886-ACT; Santa Cruz Biotechnology, Inc.), $100 \mathrm{nM}$ miR-361-5p mimic $+1 \mu \mathrm{g}$ control-plasmid or $100 \mathrm{nM}$ miR-361-5p mimic + $1 \mu \mathrm{g}$ MAP3K9-plasmid at $37^{\circ} \mathrm{C}$ for $48 \mathrm{~h}$ using Polyplus transfection reagent (Invitrogen; Thermo Fisher Scientific, Inc.) according to the manufacturer's instructions. $48 \mathrm{~h}$ after the transfection procedure was completed, subsequent experiments were performed.

Reverse transcription-quantitative PCR (RT-qPCR) assay. Total RNA was extracted from cells using TRIzol ${ }^{\circledast}$ reagent (Takara Bio, Inc.) according to the manufacturer's instructions. The RNA purity was confirmed based on A260/A280 using NanoDrop 2000 (Thermo Fisher Scientific, Inc.) in accordance with the manufacturer's instructions. When RNA extraction was completed, RNA was reverse-transcribed into complementary DNA (cDNA) using a Reverse Transcription kit (Vazyme Biotech Co., Ltd.) following the manufacturer's protocol. Subsequently, cDNA was used for amplification. qPCR was performed using a SYBR Green PCR kit (Vazyme Biotech Co., Ltd.). Thermocycling conditions used for qPCR were as follows: Initial denaturation at $95^{\circ} \mathrm{C}$ for $10 \mathrm{~min}$, followed by 37 cycles of denaturation at $95^{\circ} \mathrm{C}$ for $15 \mathrm{sec}$, annealing at $55^{\circ} \mathrm{C}$ for $40 \mathrm{sec}$ and extension at $72^{\circ} \mathrm{C}$ for $34 \mathrm{sec}$. GAPDH (for mRNA) or U6 (for miRNA) were used as endogenous controls. Primer sequences used for qPCR were as follows: GAPDH forward,
5'-TTTGGTATCGTGGAAGGACTC-3' and reverse, 5'-GTA GAGGCAGGGATGATGTTCT-3'; U6 forward, 5'-CTCGCT TCGGCAGCAGCACATATA-3' and reverse, 5'-AAATAT GGAACGCTTCACGA-3'; miR-361-5p forward, 5'-ATAAAG TGCTGACAGTGCAGATAGTG-3' and reverse, 5'-TCAAGT ACCCACAGTGCGGT-3'; MAP3K9 forward, 5'-GAGTGC GGCAGGGACGTAT-3' and reverse, 5'-CCCCATAGCTCC ACACATCAC-3'. The $2^{-\triangle \Delta C q}$ method (27) was used to quantify relative gene expression. All samples required three replicates.

Western blot assay. The cells were lysed and total protein was obtained using RIPA lysis buffer (Beyotime Institute of Biotechnology). A BCA assay kit (Pierce; Thermo Fisher Scientific,Inc.) wasused toquantify the total protein.Equal amount of proteins ( $40 \mu \mathrm{g}$ per lane) were separated by $15 \%$ SDS-PAGE for $40 \mathrm{~min}$ and then transferred to PVDF membranes (EMD Millipore). The membranes were blocked at room temperature for $1.5 \mathrm{~h}$ with $5 \%$ non-fat milk and incubated with primary antibodies, namely, anti-MAP3K9 (cat. no. ab228752; 1:1,000, Abcam), anti-cleaved caspase-3 (cat. no. ab32042; 1:1,000, Abcam) or anti-caspase-3 (cat. no. ab32351; 1:1,000, Abcam) at $4{ }^{\circ} \mathrm{C}$ overnight. On the following day, the membranes were incubated with the horseradish peroxidase-conjugated secondary antibodies (cat. no. 7074; 1:2,000; Cell Signaling Technology, Inc.) for $2 \mathrm{~h}$ at room temperature. The protein bands were visualized using the enhanced chemiluminescence method (Cytiva). GAPDH (cat. no. 9485; 1:1,000, Abcam) served as the loading control for normalization. Band densities were quantified using the Gel-Pro Analyzer densitometry software (version 6.3; Media Cybernetics, Inc.).

Flow cytometry (FCM) assay. Cell apoptosis was assessed using an Annexin-V/PI Apoptosis Detection kit (Beyotime Institute of Biotechnology). Briefly, cells $\left(5 \times 10^{4}\right.$ cells per well) were plated in 6-well plates overnight. On the following day, cells were transfected with the plasmids or mimics as aforementioned. Subsequently, the cells were collected, centrifuged with low temperature at high speed $\left(1,000 \mathrm{x} \mathrm{g}\right.$ at $4^{\circ} \mathrm{C}$ for $5 \mathrm{~min}$ ) and resuspended in $100 \mu \mathrm{l}$ of FITC-binding buffer (Beyotime Institute of Biotechnology). Subsequently, $\sim 5 \mu 1$ ready-to-use Annexin V-FITC (BD Bioscience) and $5 \mu 1$ PI were added into the buffer. The cells were incubated for $30 \mathrm{~min}$ at room temperature in the dark. Annexin V-FITC and PI fluorescence were assessed using a BD FACSCalibur flow cytometer (BD Biosciences). CellQuest software (version 5.1; BD Biosciences) was used to analyze flow cytometry data.

Dual-luciferasereporterassay.Bioinformatics software analysis (TargetScan version 7.2; http://www.targetscan.org/vert_72/) was used to predict the potential targets of miR-361-5p. The results revealed the potential binding sites between miR-361-5p and MAP3K9. To confirm this, the wild-type (wt) or mutant (mut) 3'untranslated region (UTR) of MAP3K9 was cloned into the pmiRGLO vector (Promega Corporation). The recombinant plasmids were acquired using EndoFree Plasmid Maxi kit (Vazyme Biotech Co., Ltd.). Huh7 cells seeded $\left(5 \times 10^{4}\right.$ cells per well) in 24-well plates were co-transfected with miR-361-5p mimics or negative control and the mut or wt 3'-UTR of MAP3K9, together with the Renilla luciferase pRL-TK vector as a control. After transfection at $37^{\circ} \mathrm{C}$ for $48 \mathrm{~h}$, firefly and 

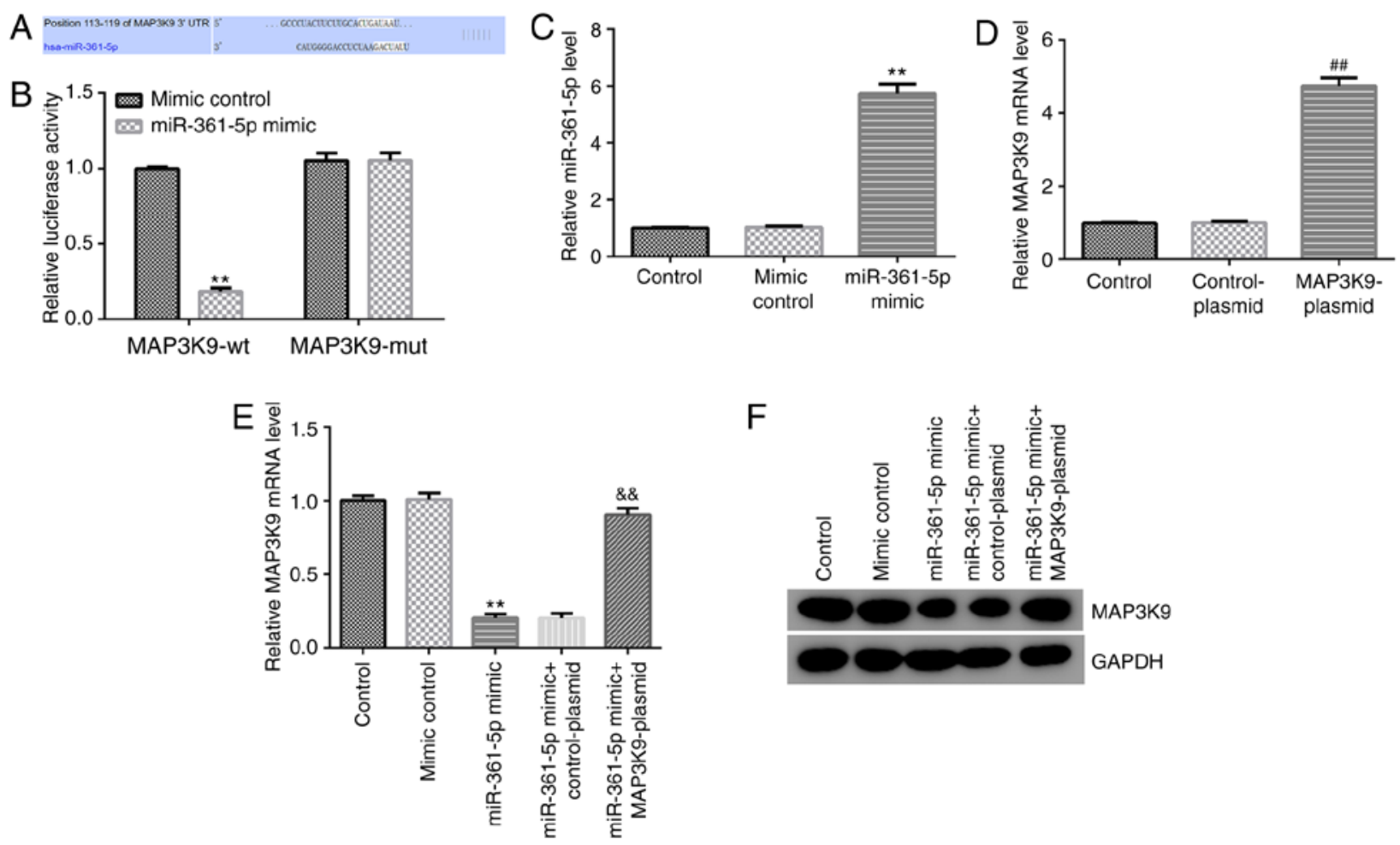

Figure 1. Association between MAP3K9 and miR-361-5p. (A) Prediction of MAP3K9 targeting by miR-361-5p using TargetScan. (B) Dual-luciferase reporter gene assays were used to verify the association between miR-361-5p and MAP3K9. (C) RT-qPCR analysis was used to detect miR-361-5p expression levels following Huh7 cell transfection with mimic control or miR-361-5p mimic. (D) RT-qPCR analysis was used to detect MAP3K9 mRNA expression levels following Huh7 cell transfection with control-plasmid or MAP3K9-plasmid. (E) RT-qPCR analysis and (F) western blot analysis was used to detect MAP3K9 mRNA and protein expression levels, respectively, following mimic and plasmid transfections. ${ }^{* *} \mathrm{P}<0.01$ vs. mimic control; ${ }^{* \#} \mathrm{P}<0.01$ vs. control-plasmid; \&\& $\mathrm{P}<0.01$ vs. miR-361-5p mimic + control-plasmid. MAP3K9, MAPK kinase kinase 9; miR, microRNA; mut, mutant; RT-qPCR, reverse transcription-quantitative PCR; UTR, untranslated region; wt, wild-type.

Renilla luciferase activity was measured using a dual-luciferase reporter assay kit (Promega Corporation). Firefly luciferase activity was normalized to Renilla luciferase activity.

DDP sensitivity assay. Different concentrations of DDP $(0.5,1,2$, $4,8,16,32$ and $64 \mu \mathrm{g} / \mathrm{ml}$ ) were used to treat Huh7 and Huh7/DDP cells $\left(5 \times 10^{4}\right.$ cell per well) at $37^{\circ} \mathrm{C}$ for $48 \mathrm{~h}$. Cell viability was then measured using MTT assays and $150 \mu \mathrm{l}$ DMSO was used to dissolve the resultant purple formazan. The $\mathrm{IC}_{50}$ value of DDP was calculated from these data. After the cells were processed according to manufacturer's instructions, $20 \mu \mathrm{l}$ MTT $(5 \mathrm{mg} / \mathrm{ml}$; Sigma-Aldrich; Merck KGaA) was added into each well and the wells were further cultured for $4 \mathrm{~h}$. The absorbance was then measured at $570 \mathrm{~nm}$. The data were analyzed and presented as the mean $\pm \mathrm{SD}$ of three independent experiments.

Statistical analysis. Statistical analyses were performed using GraphPad Prism 6.0 (GraphPad Software, Inc.). The statistical significance of the differences between groups was determined using unpaired Student's t-test or one-way ANOVAs followed by Tukey's post hoc tests. Data are expressed as the mean \pm SD from at least three independent experiments. $\mathrm{P}<0.05$ was considered to indicate statistically significant differences.

\section{Results}

Target association between miR-361-5p and MAP3K9. The present study found that miR-361-5p has hundreds of target genes, which includes MAP3K9 (Fig. 1A). As MAP3K9 is an activator upstream of the MAPK kinase/JNK signaling pathway, which plays critical roles in cell apoptosis regulation (22), and the role of MAP3K9 in HCC remain largely unclear, MAP3K9 was chosen for further investigation. The association between miR-361-5p and MAP3K9 was confirmed using dual-luciferase reporter assays. The 3'-UTR (either wt or mut) of MAP3K9 was inserted into a pmiR luciferase reporter and Huh7 cells were co-transfected with miR-361-5p mimic or mimic control and MAP3K9-wt or MAP3K9-mut. The results indicated that miR-361-5p mimic co-transfection with wt MAP3K9 3'-UTR reporter inhibited luciferase activity, but miR-361-5p mimic did not exert any effect on the reporter containing the mut MAP3K9 3'-UTR (Fig. 1B). These findings confirmed MAP3K9 as a target gene of miR-361-5p. Subsequently, Huh7 cells were transfected with mimic control, miR-361-5p mimic, control-plasmid, MAP3K9-plasmid, miR-361-5p mimic + control-plasmid or miR-361-5p mimic + MAP3K9-plasmid for $48 \mathrm{~h}$ and RT-qPCR was performed to detect the transfection efficiency. In comparison with the mimic control group, miR-361-5p increased miR-361-5p expression (Fig. 1C); and compared with the control-plasmid group, MAP3K9-plasmid increased MAP3K9 expression at the mRNA level (Fig. 1D). Furthermore, miR-361-5p markedly decreased MAP3K9 protein expression and significantly decreased MAP3K9 mRNA levels in Huh7 cells, with this reduction being reversed by the MAP3K9-plasmid (Fig. 1E and F). Therefore, MAP3K9 

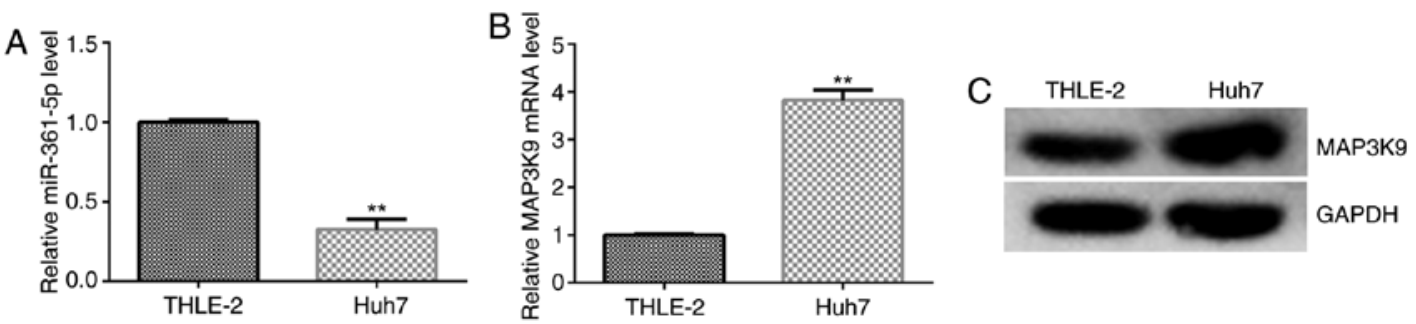

Figure 2. miR-361-5p is downregulated and MAP3K9 is upregulated in hepatocellular carcinoma cells. (A) RT-qPCR analysis was used to detect (A) miR-361-5p and (B) MAP3K9 mRNA expression levels in THLE-2 and Huh7 cells. (C) Western blot analysis was used to detect MAP3K9 protein expression levels in THLE-2 and Huh7 cells. ${ }^{* *}$ P<0.01 vs. THLE-2 cells. MAP3K9, MAPK kinase kinase 9; miR, microRNA; RT-qPCR, reverse transcription-quantitative PCR.
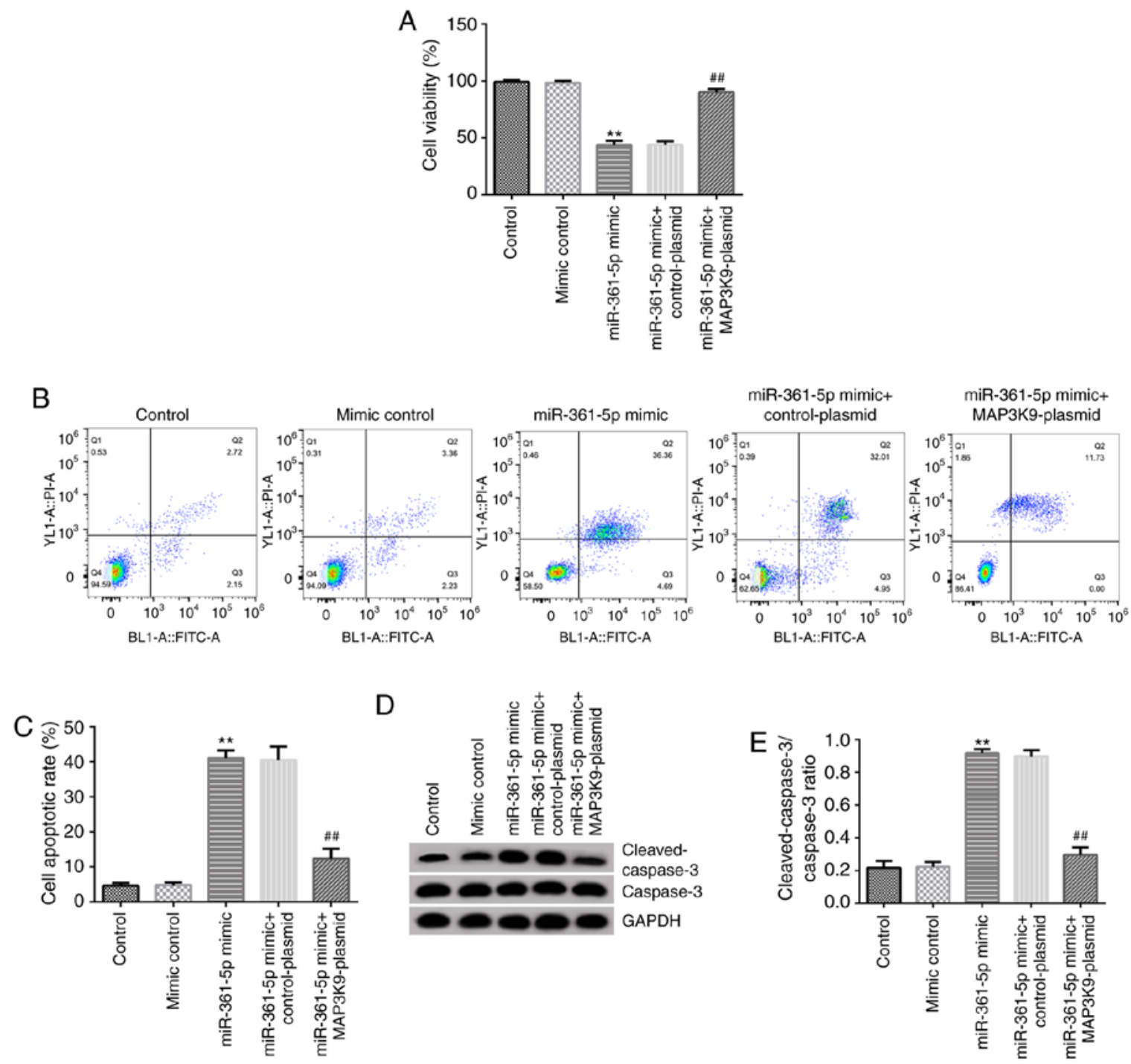

Figure 3. MAP3K9 significantly reverses miR-361-5p-induced apoptosis of hepatocellular carcinoma cells. (A) MTT analysis was used to measure cell viability following Huh7 cell transfection with mimic control, miR-361-5p mimic, miR-361-5p mimic + control-plasmid or miR-361-5p mimic + MAP3K9-plasmid for 48 h. (B) Flow cytometry analysis was used to measure cell apoptosis. (C) Cell apoptosis rate was quantified using GraphPad 6.0. (D) Western blot assays were used to examine the protein expression levels of cleaved caspase-3 and caspase-3. (E) The cleaved caspase-3/caspase-3 ratio was quantified using Gel-Pro Analyzer densitometry software. ${ }^{* *} \mathrm{P}<0.01$ vs. mimic control; ${ }^{\# \#} \mathrm{P}<0.01$ vs. miR-361-5p mimic + control-plasmid. MAP3K9, MAPK kinase kinase 9; miR, microRNA.

expression was negatively regulated by miR-361-5p in Huh7 cells.

Expression levels of miR-361-5p and MAP3K9 in HCC cells. Subsequently, the expression levels of miR-361-5p and
MAP3K9 in normal human hepatocytes (THLE-2) and Huh7 HCC cells was examined. RT-qPCR indicated that compared with the THLE- 2 cells, the miR-361-5p expression levels were lower in Huh7 cells (Fig. 2A), while the MAP3K9 expression levels were markedly higher (Fig. 2B and C). 

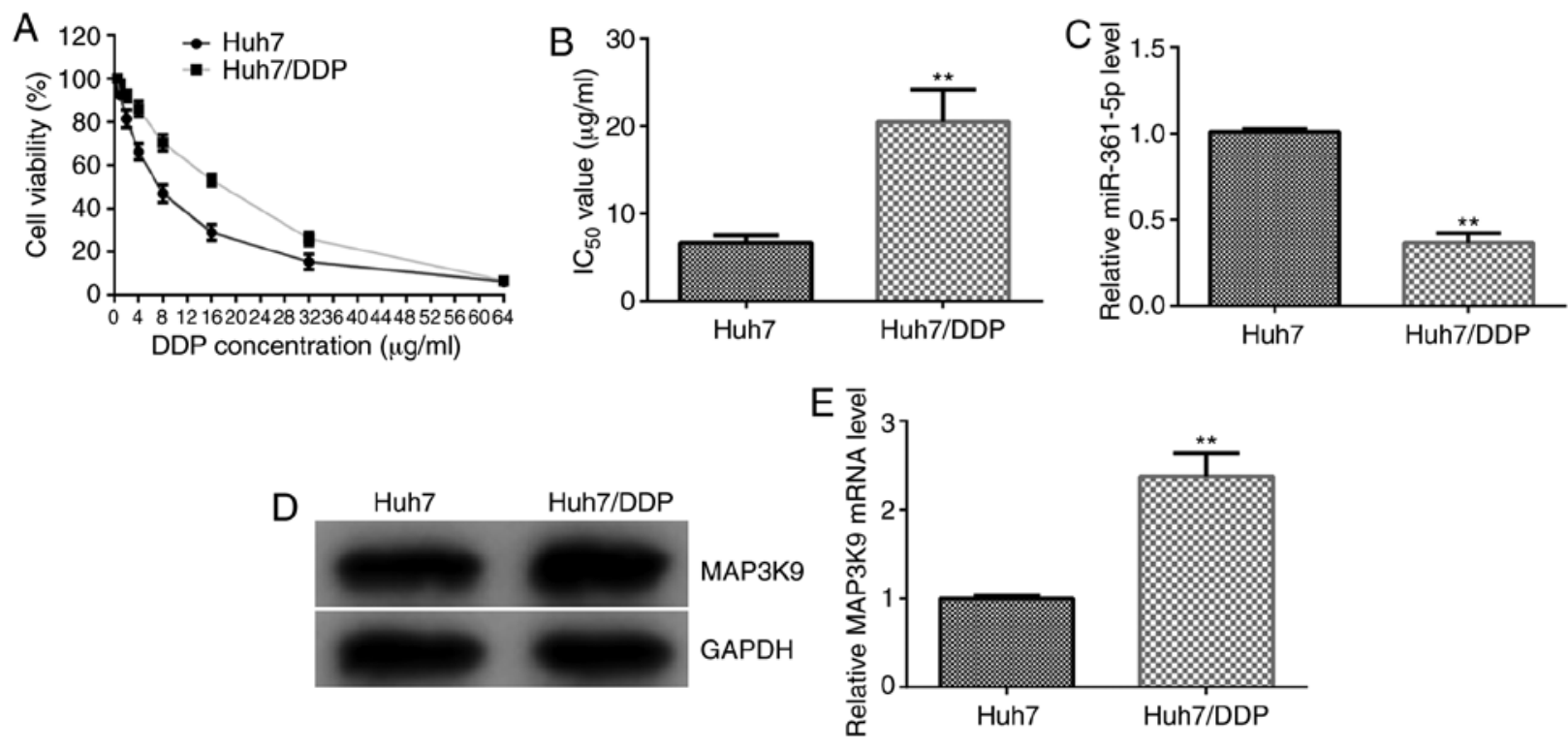

Figure 4. miR-361-5p is downregulated and MAP3K9 is upregulated in Huh7/DDP cells. (A) MTT analysis was used to measure the viability of Huh7/DDP and Huh7 cells after DDP treatment. (B) The $\mathrm{IC}_{50}$ values of DDP on Huh7/DDP and Huh7 cells was calculated using GraphPad 6.0. (C) RT-qPCR was used to determine miR-361-5p expression in Huh7/DDP and Huh7 cells. (D) Western blot assays and (E) RT-qPCR were used to measure the protein and mRNA expression levels, respectively, of MAP3K9 in Huh7/DDP and Huh7 cells. ${ }^{* *} \mathrm{P}<0.01$ vs. Huh7 cells. DDP, cisplatin; RT-qPCR, reverse transcription-quantitative PCR.

Effects of miR-361-5p on the viability and apoptosis of HCC cells. The effect of miR-361-5p and MAP3K9 on the viability and apoptosis of Huh7 cells was next investigated. Huh7 cells were transfected with mimic control, miR-361-5p mimic, miR-361-5p mimic + control-plasmid or miR-361-5p mimic + MAP3K9-plasmid and a series of experiments were performed after $48 \mathrm{~h}$. MTT assays demonstrated that compared with the mimic control group, miR-361-5p reduced Huh7 cell viability (Fig. 3A). FCM assay indicated that miR-361-5p promoted Huh7 cell apoptosis (Fig. 3B and C). In addition, western blotting demonstrated that miR-361-5p increased cleaved caspase-3 expression (Fig. 3D) and increased the ratio of cleaved caspase-3/caspase-3 (Fig. 3E). All these effects were notably reversed by co-transfection with the MAP3K9-plasmid.

Expression levels of miR-361-5p in Huh7/DDP cells. DDP is currently the most widely used antitumor drug in clinical practice; however, due to cancer cell resistance to DDP, novel treatment options for $\mathrm{HCC}$ are needed. In the present study, the DDP-resistant cell line Huh7/DDP was established by exposing parental Huh7 cells to increasing concentrations of DDP for 12 months. Subsequently, after treating Huh7 and Huh7/DDP cells with various concentrations of DDP $(0.5,1,2$, $4,8,16,32$ and $64 \mathrm{mg} / \mathrm{ml}$ ) for $48 \mathrm{~h}$, the viability of Huh7/DDP cells was found to be higher compared with that of Huh7 cells at $4,8,16,32 \mathrm{mg} / \mathrm{ml}$ DDP treatment (Fig. $4 \mathrm{~A}$ ). The $\mathrm{IC}_{50}$ value of DDP on Huh7/DDP cells was higher compared with that of Huh7 cells (Fig. 4B). Subsequently, RT-qPCR demonstrated that the expression levels of miR-361-5p in Huh7/DDP cells was lower compared with that in the Huh7 cells (Fig. 4C). Moreover, it was found that the protein expression levels of MAP3K9 were increased in Huh7/DDP cells compared with the Huh7 cells (Fig. 4D). RT-qPCR also demonstrated that the mRNA expression levels of MAP3K9 in Huh7/DDP cells was significantly higher compared with that in the Huh7 cells (Fig. 4E)

MAP3K9 is negatively regulated by miR-361-5p in Huh7/DDP cells. Huh7/DDP cells were next transfected with mimic control, miR-361-5p mimic, control-plasmid, MAP3K9-plasmid, miR-361-5p mimic + control-plasmid or miR-361-5p mimic + MAP3K9-plasmid for $48 \mathrm{~h}$. The transfection efficiency of MAP3K9 and miR-361-5p was detected after $48 \mathrm{~h}$. In comparison with the mimic control group, miR-361-5p increased miR-361-5p expression levels in Huh7/DDP cells (Fig. 5A). In comparison with the control-plasmid group, MAP3K9-plasmid transfection increased MAP3K9 mRNA expression levels in Huh7/DDP cells (Fig. 5B). In comparison with the mimic control group, miR-361-5p significantly reduced MAP3K9 mRNA expression levels and notably reduced the protein expression levels in Huh7/DDP cells. This reduction was reversed following transfection with the MAP3K9-plasmid (Fig. 5C and D).

Effect of miR-361-5p on the sensitivity of Huh7/DDP cells. Finally, the effects of MAP3K9 and miR-361-5p on the viability of Huh7/DDP cells was examined. The MTT assay indicated that miR-361-5p mimic significantly decreased Huh7/DDP cell viability and reduced the $\mathrm{IC}_{50}$ value of DDP on Huh7/DDP cells, whereas these effects were reversed by the MAP3K9-plasmid (Fig. 6A and B). Compared with the mimic control group, miR-361-5p mimic increased apoptosis of Huh7/DDP cells (Fig. 6C and D); moreover, miR-361-5p mimic increased cleaved caspase-3 protein expression levels and the cleaved caspase-3/caspase-3 ratio in Huh7/DDP cells. However, all these effects were reversed by transfection with the MAP3K9-plasmid. 

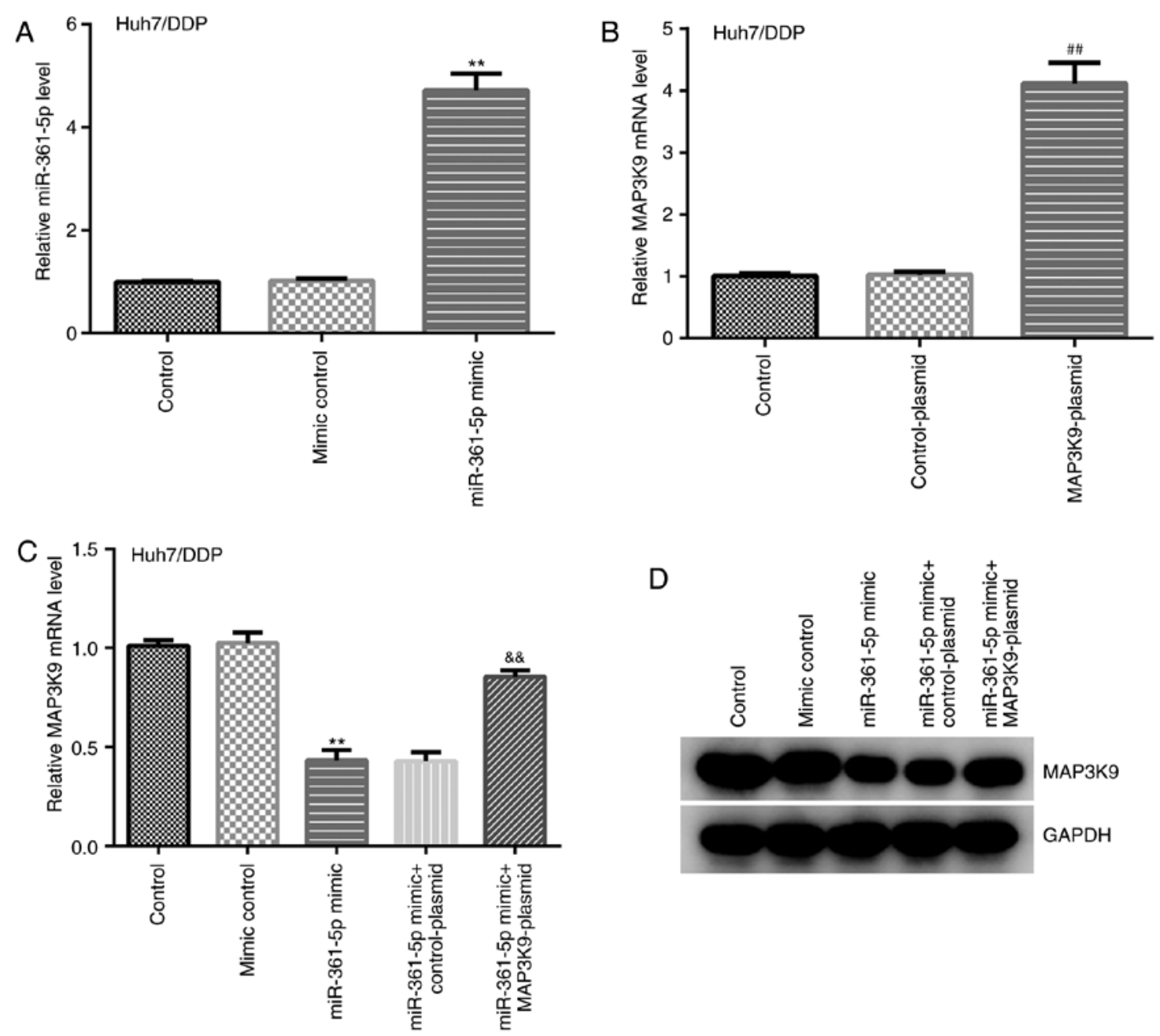

Figure 5. MAP3K9 is negatively regulated by miR-361-5p in Huh7/DDP cells. (A) RT-qPCR analysis was used to determine miR-361-5p expression levels in the mimic control and miR-361-5p mimic groups. (B) RT-qPCR analysis was used to determine MAP3K9 mRNA expression levels in the control-plasmid and MAP3K9-plasmid groups. (C) RT-qPCR analysis was used to determine MAP3K9 mRNA expression levels and (D) western blot assays were used to determine MAP3K9 protein expression levels in Huh7/DDP cells following the various treatments. ${ }^{\text {** }} \mathrm{P}<0.01 \mathrm{vs}$. mimic control; ${ }^{\# \#} \mathrm{P}<0.01 \mathrm{vs}$. control-plasmid; ${ }^{\&} \mathrm{P}<0.01$ vs. miR-361-5p mimic + control-plasmid. MAP3K9, MAPK kinase kinase 9; miR, microRNA; RT-qPCR, reverse transcription-quantitative PCR.

\section{Discussion}

The development of HCC represents a major health concern worldwide (2). Despite advances in medical techniques in recent years, the mortality rate of $\mathrm{HCC}$ remains high due to the difficulty of early diagnosis, relative lack of therapeutic targets and poor postoperative recovery $(7,8)$. Following surgical resection, the number of patients with $\mathrm{HCC}$ who survive for 1 year has been reported to be $>80 \%$, but the 5 -year survival rate decreases to $>50 \%$ (28). Therefore, there is a continued need to identify novel targets for the treatment of patients with HCC. Previous studies have demonstrated that miR-361-5p expression is downregulated in HCC cells $(19,29)$. The present study also demonstrated that miR-361-5p was downregulated in Huh7 cells. Additionally, Zhang et al (30) reported that miR-361-5p expression is decreased in lung cancer, whereas Li et al (31) demonstrated that the expression miR-361-5p was low in papillary thyroid carcinoma.

The present study demonstrated that there was an interaction site between miR-361-5p and MAP3K9. RT-qPCR revealed that was a negative association between MAP3K9 expression and miR-361-5p expression in Huh7 cells. MAP3K9 is a member of a mixed-lineage family of kinases, consisting of the SH3 domain, the Ser/Thr kinase domain and the Cdc42/Rac interaction binding domain $(32,33)$. Moreover, this kinase has also been found to be an upstream activator of the JNK and ERK signaling pathways (34). A number of studies have demonstrated that MAP3K9 is a target of several miRNAs, including miR-574-5p (35), miR-490-5p (36) and miR-15a (37). However, the role of MAP3K9 in HCC remains unclear. The results of the present study indicated that MAP3K9 was able to significantly reverse miR-361-5p-induced apoptosis of HCC cells.

HCC cells commonly become resistant to DDP, resulting in chemotherapy failure (38). The present data and those of previous reports demonstrated that miR-361-5p significantly inhibited the proliferation and induced apoptosis of HCC cells $(19,20)$. However, only the ratio of cleaved caspase- 3 and caspase-3 (apoptosis-related proteins) were used to measure cell apoptosis in the present study. To make the results more convincing, changes in expression levels of apoptosis-related proteins Bax and Bcl-2 should also be investigated, which was a limitation of the present study. To the best of our knowledge, prior to the present study, the exact role of miR-361-5p in HCC chemoresistance remained elusive. Therefore, the present 

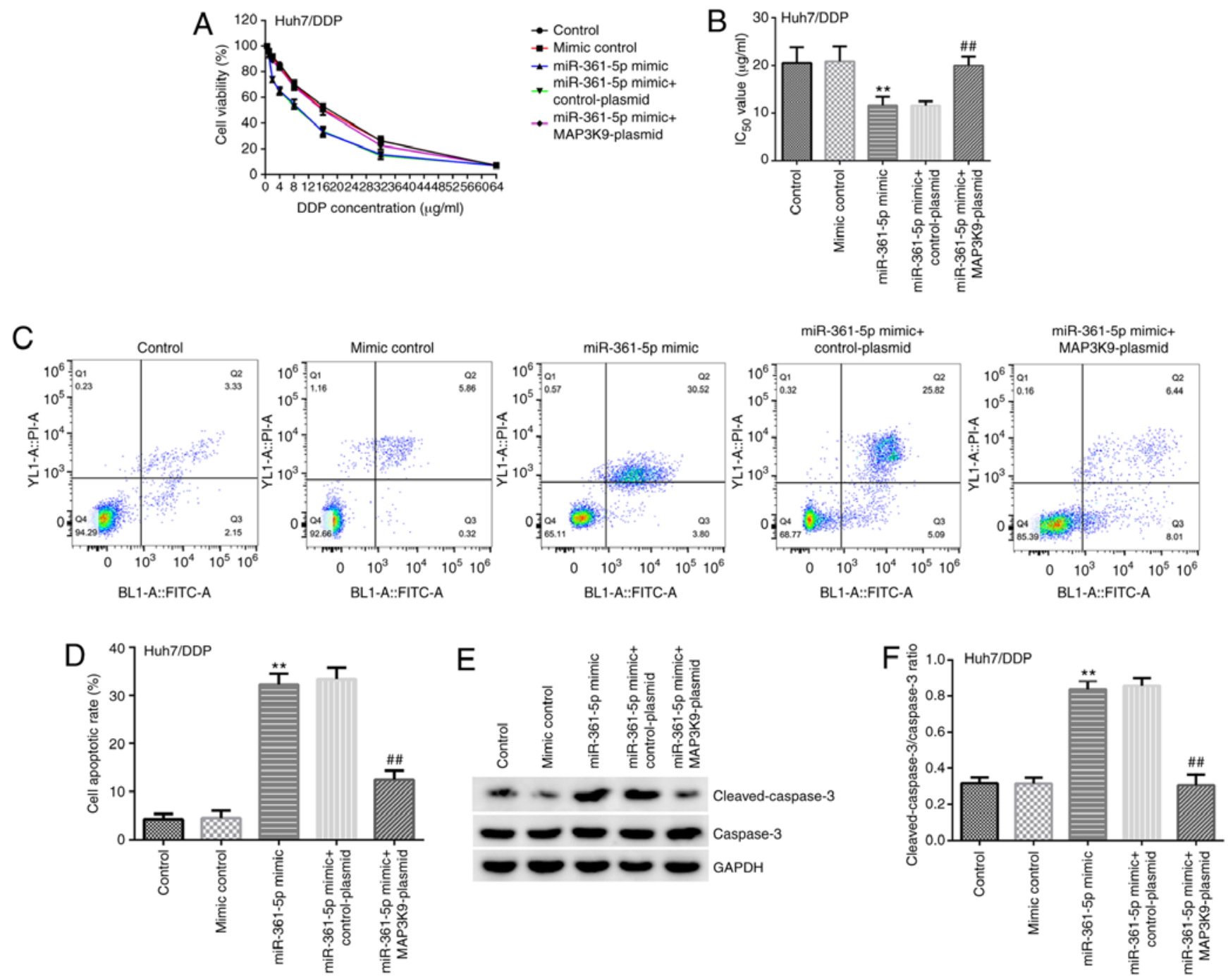

Figure 6. MAP3K9 significantly reverses the miR-361-5p-induced increase in the sensitivity of Huh7/DDP cells to DDP and miR-361-5p-induced cell apoptosis. Huh7/DDP cells were transfected with mimic control, miR-361-5p mimic, control-plasmid, MAP3K9-plasmid, miR-361-5p mimic + control-plasmid or miR-361-5p mimic + MAP3K9-plasmid for 48 h. (A) MTT assays were used to determine cell viability. (B) The $\mathrm{IC}_{50}$ value of DDP on Huh7/DDP cells in the various treatment groups. (C) Flow cytometry was used to determine Huh7/DDP cell apoptosis. (D) The cell apoptotic rates were calculated using GraphPad 6.0. (E) Western blot assays were used to investigate cleaved caspase-3 and caspase-3 protein expression levels and the (F) cleaved caspase-3/caspase-3 ratio was calculated in the various treatment groups. ${ }^{* *} \mathrm{P}<0.01 \mathrm{vs}$. mimic control; ${ }^{\# \#} \mathrm{P}<0.01 \mathrm{vs}$. miR-361-5p mimic + control-plasmid. DDP, cisplatin; MAP3K9, MAPK kinase kinase 9; miR, microRNA.

study used DDP-resistant HCC cells to study the function and potential mechanism of action of miR-361-5p. Huh7/DDP and Huh7 cells were then treated with various concentrations of DDP and the $\mathrm{IC}_{50}$ value of Huh7/DDP cells was found to be higher compared with that of Huh7 cells. In addition, the expression levels of miR-361-5p were lower in Huh7/DDP cells compared with that in Huh7 cells. Finally, MAP3K9 significantly reversed the miR-361-5p-induced increased sensitivity of Huh7/DDP cells to DDP.

It should be noted; however, that the present study was only a preliminary in vitro investigation into the role of miR-361-5p in HCC chemotherapy resistance. To identify the role of miR-361-5p/MAP3K9 in HCC chemotherapy resistance, further studies are required.Forexample, the role of miR-361-5p/MAP3K9 on the Huh7 and Huh7/DDP cell migratory, invasive and clone formation abilities should be explored using wound healing, Transwell and clone formation assays. Furthermore, the effect of miR-361-5p on HCC chemotherapy resistance should be investigated in vivo. Moreover, the expression of miR-361-5p and MAP3K9 in HCC cancer tissues need to be explored.

In conclusion, the findings of the present study demonstrated that miR-361-5p induces HCC cell apoptosis and enhances drug sensitivity by targeting MAP3K9.

\section{Acknowledgements}

Not applicable.

\section{Funding}

No funding was received.

\section{Availability of data and materials}

The datasets used and/or analyzed during the current study are available from the corresponding author on reasonable request. 


\section{Authors' contributions}

QR and XX contributed to study design, data collection, statistical analysis, data interpretation and manuscript preparation. XL, QZ, XZ and ZR contributed to data collection and data interpretation. HX contributed to data collection, statistical analysis and manuscript preparation. QR and XX confirm the authenticity of all the raw data. All authors read and approved the final manuscript.

\section{Ethics approval and consent to participate}

Not applicable.

\section{Patient consent for publication}

Not applicable.

\section{Competing interests}

The authors declare that they have no competing interests.

\section{References}

1. Clark T, Maximin S, Meier J, Pokharel S and Bhargava P: Hepatocellular carcinoma: Review of epidemiology, screening, imaging diagnosis, response assessment, and treatment. Curr Probl Diagn Radiol 44: 479-486, 2015.

2. Wallace MC, Preen D, Jeffrey GP and Adams LA: The evolving epidemiology of hepatocellular carcinoma: A global perspective. Expert Rev Gastroenterol Hepatol 9: 765-779, 2015.

3. Oliveri RS, Wetterslev J and Gluud C: Hepatocellular carcinoma. Lancet 380: 470-471, 2012.

4. Bray F, Ferlay J, Soerjomataram I, Siegel RL, Torre LA and Jemal A: Global cancer statistics 2018: GLOBOCAN estimates of incidence and mortality worldwide for 36 cancers in 185 countries. CA Cancer J Clin 68: 394-424, 2018,

5. Siegel RL, Miller KD and Jemal A: Cancer statistics, 2019. CA Cancer J Clin 69: 7-34, 2019.

6. Farazi PA and DePinhO RA: Hepatocellular carcinoma pathogenesis: From genes to environment. Nat Rev Cancer 6: 674-687, 2006.

7. Hartke J, Johnson M and Ghabril M: The diagnosis and treatment of hepatocellular carcinoma. Semin Diagn Pathol 34: 153-159, 2017.

8. Sinn DH, Choi GS, Park HC, Kim JM, Kim H, Song KD, Kang TW, Lee MW, Rhim H, Hyun D, et al: Multidisciplinary approach is associated with improved survival of hepatocellular carcinoma patients. PLoS One 14: e0210730, 2019.

9. Giaccone G: Clinical perspectives on platinum resistance. Drugs 59 (Suppl 4): S9-S17; discussion 37-8, 2000.

10. Galanski M: Recent developments in the field of anticancer platinum complexes. Recent Pat Anticancer Drug Discov 1: 285-295, 2006.

11. Koberle B, Tomicic MT, Usanova S and Kaina B: Cisplatin resistance: Preclinical findings and clinical implications. Biochim Biophys Acta 1806: 172-182, 2010.

12. Johnsson P, Lipovich L, Grander D and Morris KV: Evolutionary conservation of long non-coding RNAs; sequence, structure, function. Biochim Biophys Acta 1840: 1063-1071, 2014.

13. Bartel DP: MicroRNAs: Genomics, biogenesis, mechanism, and function. Cell 116: 281-297, 2004.

14. Mo YY: MicroRNA regulatory networks and human disease. Cell Mol Life Sci 69: 3529-3531, 2012.

15. Laffont B and Rayner KJ: MicroRNAs in the pathobiology and therapy of atherosclerosis. Can J Cardiol 33: 313-324, 2017.

16. Ambros V: The functions of animal microRNAs. Nature 431: 350-355, 2004.

17. Wang Q, Yang X, Zhou X, Wu B, Zhu D, Jia W, Chu J, Wang J, Wu J and Kong L: miR-3174 promotes proliferation and inhibits apoptosis by targeting FOXO1 in hepatocellular carcinoma. Biochem Biophys Res Commun 526: 889-897, 2020.
18. Wang J, Lu L, Luo Z, Li W, Lu Y, Tang Q and Pu J: miR-383 inhibits cell growth and promotes cell apoptosis in hepatocellular carcinoma by targeting IL-17 via STAT3 signaling pathway. Biomed Pharmacother 120: 109551, 2019.

19. Sun JJ, Chen GY and Xie ZT: MicroRNA-361-5p inhibits cancer cell growth by targeting CXCR6 in hepatocellular carcinoma. Cell Physiol Biochem 38: 777-785, 2016.

20. Cui W, Li Y, Xu K, Chen G, Lu X, Duan Q and Kang Z: miR-361-5p inhibits hepatocellular carcinoma cell proliferation and invasion by targeting VEGFA. Biochem Biophys Res Commun 479: 901-906, 2016.

21. Gong D, Feng PC, Ke XF, Kuang HL, Pan LL, Ye Q and Wu JB: Silencing long non-coding RNA LINC01224 inhibits hepatocellular carcinoma progression via microRNA-330-5p-induced inhibition of CHEK1. Mol Ther Nucleic Acids 19: 482-497, 2020.

22. Fawdar S, Trotter EW,Li Y, Stephenson NL, Hanke F, Marusiak AA, Edwards ZC, Ientile S, Waszkowycz B, Miller CJ and Brognard J: Targeted genetic dependency screen facilitates identification of actionable mutations in FGFR4, MAP3K9, and PAK5 in lung cancer. Proc Natl Acad Sci USA 110: 12426-12431, 2013.

23. Munshi A and Ramesh R: Mitogen-activated protein kinases and their role in radiation response. Genes Cancer 4: 401-408, 2013.

24. Nie F, Liu TM, Zhong L, Yang X, Liu Y, Xia H, Liu X, Wang X, Liu Z, Zhou L, et al: MicroRNA-148b enhances proliferation and apoptosis in human renal cancer cells via directly targeting MAP3K9. Mol Med Rep 13: 83-90, 2016.

25. Zhao F, LV J, Gan H, Li Y, Wang R, Zhang H, Wu Q and Chen Y: miRNA profle of osteosarcoma with CD117 and stro-1 expression: miR-1247 functions as an onco-miRNA by targeting MAP3K9. Int J Clin Exp Pathol 8: 1451-1458, 2015.

26. Liu Z, Dang C, Xing E, Zhao M, Shi L and Sun J: Overexpression of CASC2 improves cisplatin sensitivity in hepatocellular carcinoma through sponging miR-222. DNA Cell Biol 38: 1366-1373, 2019.

27. Livak KJ and Schmittgen TD: Analysis of relative gene expression data using real-time quantitative PCR and the 2(-Delta Delta C(T)) method. Methods 25: 402-408, 2001.

28. Margarit C, Escartín A, Castells L, Vargas V, Allende E and Bilbao I: Resection for hepatocellular carcinoma is a good option in Child-Turcotte-Pugh class A patients with cirrhosis who are eligible for liver transplantation. Liver Transpl 11: 1242-1251, 2005.

29. Cheng Y, Qiu L, He GL, Cai L, Peng BJ, Cao YL and Pan MX: MicroRNA-361-5p suppresses the tumorigenesis of hepatocellular carcinoma through targeting WT1 and suppressing WNT/ $\beta$-cadherin pathway. Eur Rev Med Pharmacol Sci 23: 8823-8832, 2019.

30. Zhang S, Liu Z, Wu L and Wang Y: miR-361 targets Yes-associated protein (YAP) mRNA to suppress cell proliferation in lung cancer. Biochem Biophys Res Commun 492: 468-473, 2017.

31. Li R, Dong B, Wang Z, Jiang T and Chen G: MicroRNA-361-5p inhibits papillary thyroid carcinoma progression by targeting ROCK1. Biomed Pharmacother 102: 988-995, 2018.

32. Slattery ML, Lundgreen A and Wolff RK: MAP kinase genes and colon and rectal cancer. Carcinogenesis 33: 2398-2408, 2012.

33. Stark MS, Woods SL, Gartside MG, Bonazzi VF, Dutton-RegesterK, Aoude LG, Chow D, Sereduk C, Niemi NM, Tang N, et al: Frequent somatic mutations in MAP3K5 and MAP3K9 in metastatic melanoma identifed by exome sequencing. Nat Genet 44: 165-169, 2011.

34. Thompson NA, Haefliger JA, Senn A, Tawadros T, Magara F, Ledermann B, Nicod P and Waeber G: Islet-brain1/JNK-interacting protein-1 is required for early embryogenesis in mice. J Biol Chem 276: 27745-27748, 2001.

35. Liu Y, Hou J, Zhang M, Seleh-Zo E, Wang J, Cao B and An X: Circ-016910 sponges miR-574-5p to regulate cell physiology and milk synthesis via MAPK and PI3K/AKT-mTOR pathways in GMECs. J Cell Physiol 235: 4198-4216, 2020.

36. Abdeyrim A, Cheng X, Lian M and Tan Y: miR-490-5p regulates the proliferation, migration, invasion and epithelial-mesenchymal transition of pharyngolaryngeal cancer cells by targeting mitogen-activated protein kinase kinasekinase 9. Int J Mol Med 44: 240-252, 2019.

37. Cai P, Yang T, Jiang X, Zheng M, Xu G and Xia J: Role of miR-15a in intervertebral disc degeneration through targeting MAP3K9. Biomed Pharmacother 87: 568-574, 2017.

38. Zhang X, Li J and Yan M: Targeted hepatocellular carcinoma therapy: Transferrin modified, self-assembled polymeric nanomedicine for co-delivery of cisplatin and doxorubicin. Drug Dev Ind Pharm 42: 1590-1599, 2016.

This work is licensed under a Creative Commons Attribution-NonCommercial-NoDerivatives 4.0 International (CC BY-NC-ND 4.0) License. 